\title{
Article \\ Blockchain-based Rice Supply Chain Traceability to Ensure Rice quality and food safety
}

\author{
Qi Tao ${ }^{1}{ }^{\mathbb{D}}$, Adnan Iftekhar ${ }^{1}$, Zhenyu Cai ${ }^{1}$, and Xiaohui Cui ${ }^{1 *}$ \\ 1 Key Laboratory of Aerospace Information Security and Trusted Computing, Ministry of Education, School \\ of Cyber Science and Engineering, Wuhan University, Wuhan, 430072, China \\ * Correspondence: xcui@whu.edu.cn
}

\begin{abstract}
Rice is the grain feeds more than half of the world population. It is an important staple food provides twenty percent of the calories consumed worldwide. Food safety affects the health of consumers. Establishing an effective traceability system is one of the primary measures to protect rice quality and food safety. Blockchain-based traceability system has the characteristics of data tamper-proofing, decentralization, and co-governance. This paper proposed a rice traceability scheme based on blockchain technology that selects the critical nodes in the rice industry chain to construct a blockchain network to achieve transparent data sharing. It is meaningful for both enterprises and consumers. It can let enterprises get the real quality information of rice and related raw materials. Consumers can get the true information of rice and rice products to buy safe and healthy food. The scheme is proved feasible by simulating the traceability of the rice supply chain.
\end{abstract}

Keywords: Blockchain; Rice; Supply chain traceability; Quality control

\section{Introduction}

Rice is the grain that feeds more than half of the world population. A lot of rice varieties of different species exist with yields ranging from 1-3t/ha to $5 t /$ ha [1]. In the process of selecting better cultivars, a special role is also played by the use of radiationinduced mutations. In recent years, with the exposure of poisoned rice, golden rice, and cadmium rice and frequent other rice problems seriously harmed the consumer's health and has affected market order stability. It is a challenge to distinguish rice quality from traditional methods, leading to a severe market phenomenon "Bad money drives out good." Therefore, society is getting more and more concerned about rice quality and food safety. The demand for the authenticity of the information on rice source and production process is also increasing rapidly.

The rice quality traceability system provides an effective solution to protect rice quality and safety. It conduces the rice industry structure's optimization and selects the characteristic information related to the traceability target. It determines the stability and reliability of the traceability information [2]. Over the years, researchers have made many significant contributions to the field of food traceability. These achievements also include the following significant methods. The near-infrared spectroscopy [3] but the limitations of technology, modeling methods, and algorithms made it challenging to popularize and applicable. The Isotope labeling traceability is good, but the cost is high, and the efficiency is low [4]. DNA traceability [5][6] process of food processing usually leads to the degradation and fragmentation of DNA. However, it would affect identification and traceability. The Internet of things label traceability methods gained popularity in the market due to the characteristics of efficiency and convenience [7].

However, there is a serious phenomenon that a small number of central database organizations control most of the valuable information in the traditional centralized label traceability system. It is easy to hinder the information flow and generate an information island, which will form a high-cost and inefficient operation status. Besides, the information providers can selectively shield or even tamper with information that is not favorable. At the same time, the consumer can hardly identify it with the naked eye. If the rice industry information is shared, the development of the food industry 
will experience significant benefits. It will lead to research on optimizing the industry chain and improving food traceability and anti-counterfeit. The blockchain technology can build a trusted way with different organizations to support data sharing and ensure the data reliability [8]. It is meaningful for all participants in the rice supply chain, as follows.

- It allows consumers to obtain the true information of the product to buy healthy food $[9,10]$.

- The data sharing in rice industry enables enterprises and farms to understand the real demand for their products in different region of the market, which is helpful to effectively avoid food waste and economic lost due to goods excess inventory [11].

- The existing market supervision system is not intelligent enough, and the government departments are facing severe market supervision pressure. They are eager to relieve the market supervision pressure through data analysis. Blockchain-based traceability scheme provides the possibility for the intelligent supervision of the market [12].

Some scholars have devoted to theoretical researches on blockchain technology. Stadtler et al. discussed supply chain management's critical factors, general strategies, and design directions [13]. Huckle et al. proposed blockchain traceability for fake news [14]. Kim et al. analyzed blockchain technology's advantages in traceability projects with the Internet of Things and blockchain technology [15]. The author summarized and analyzed supply chain management techniques in great detail in his article [16]. Storøy et al. proposed the TraceFood framework to facilitate data exchange between different traceability systems and designed a common standard (TraceCore XML) to exchange electronic trace information [17]. Adnan et al. design and implement a system for securing chain of custody in food supply chain based on an enterprise ready blockchian platform $[9,18]$. Sun Zhiguo et al. explained that blockchain technology is the trend of agricultural traceability systems in the future [19]. Cocco et al. studied the opportunities and challenges of blockchain technology in the banking field. It helped optimize the global financial infrastructure and achieve sustainable development with higher efficiency than traditional systems [20]. With the excellent characteristic of blockchain technology, this paper researched and constructed a rice quality traceability system of the entire industry chain. It established a trust relationship among various industry chain entities with the mathematical method feature of blockchain, eliminating third-party participation. Moreover, the consensus tamper-proofing feature enables us to virtually guarantee the data's reliability in the system [8].

The rest of this paper is as follows: Section 2 introduces the blockchain technology and the traceability system in rice supply chain. Section 3 introduces construction of a rice traceability scheme. Section 4 describes the rice traceability system implementation. The conclusion is in section 5 .

\section{Preliminaries}

It first introduces the blockchain technology and analyzes the reasons of selecting consortium blockchain to construct the system. Then, it describes the traceability system in rice industry chain.

\subsection{Blockchain Technology}

Blockchain technology has some advantage characteristics of decentralization, tamper-proof, co-governance, and anonymity [21]. It can avoid data being corrupted by internal administrators and external hackers among the processes such as storage, transmission, and display, with blockchain features of decentralization and tamper-proof. The characteristics of co-governance and consensus enabled any participants to trust the system's data, facilitating the popularity of system application. The anonymity in blockchain technology would protect consumers' privacy where any user privacy information was stored anonymously [22]. When quality and safety accidents occur, 
the personal information of producers and consumers can be protected. It enabled to effectively prevent the occurrence of mass incidents and the excessive spread of cyber violence.

According to different application scenarios, the blockchain divided into public blockchain, consortium blockchain, and private blockchain [8] [13][18][23].

- Public blockchain. It allowed allows any node to join and or quit the network freely. All nodes have equal rights in the chain, with collective maintenance of one chain by the whole network, making it a decentralized network. Regardless of PoW and PoS algorithms' adoption, the performance is relatively slow due to the entire network's transaction verification requirements. A typical example is Bitcoin.

- Private blockchain. The permission of each node in the private blockchain is controlled internally system. Its only service to single entities or individuals. The entire network is easy to maintain and has excellent privacy.

- Consortium blockchain. Each node can be added or logged out of the blockchain network after being authorized by the entity organization. Each node can have different functions in the network. The entire network is organized as a unit to form a related alliance to maintain the blockchain network's operation jointly. The transaction speed is faster than the public chain, and each node has low accounting costs and can be regulated. A typical example is Hyperledger Fabric.

Compared with public blockchain or private blockchain [8], the consortium blockchain can satisfy multi-scene applications and have several advantages as follows.

- Better performance than the public blockchain.

- It supports the identity certificate service, which can provide higher security and better controllability.

- It not only meets the needs of the food industry but also fulfills the regulatory requirements of the management agencies for rice quality safety.

- Only enterprise nodes authorized by certificates can join the blockchain network, which guarantees each node's reliability in the network and the security of interenterprise transactions.

Based on these features mentioned, the consortium blockchain was used in the construction of rice quality traceability system.

\section{Rice Traceability Scheme}

Traceability and tracking are reciprocal processes. Traceability is a process of identifying a specified product source from upstream to downstream in the industrial chain. Tracking is the opposite of the industrial chain. A traceability system should support traceability and tracking [24]. In order to construct a rice traceability system, the process should include the following steps.

- Analyze the structure of the rice industry chain and record the information of logistics and information flow processes.

- Study the essential natural fingerprint information used for rice traceability.

- $\quad$ Select feature information associated with the traceability target.

- $\quad$ Ensure the stability and reliability of traceability information.

Combined with the previous analysis of the key nodes in the food supply chain[8], this paper chooses four main links in the rice industry chain as information collection nodes illustrated in Figure 1. Suppose the products in circulation from upstream to downstream. Any records in downstream nodes can be traced to the relevant upstream node details. Even it can be traced back to the details of the planting. The main functions of each node are as follows:

- $\quad$ Planting node: Collect various data in planting link; it includes information of varieties, planting areas and soil $\mathrm{pH}$, water quality, chemical fertilizers, and pesticides. 


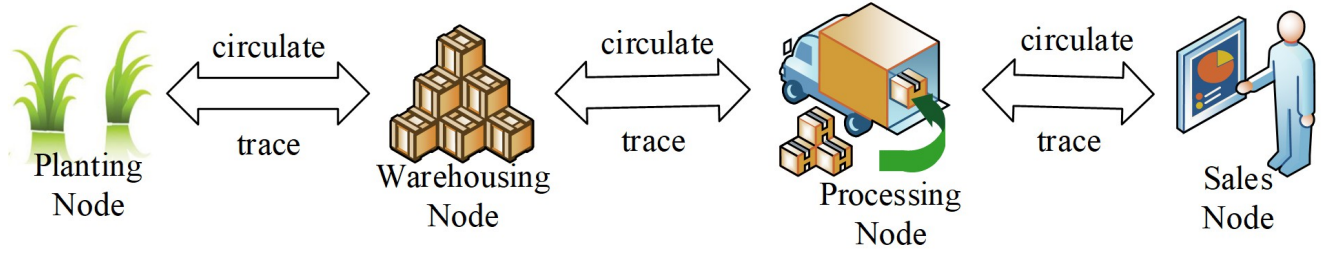

Figure 1. Traceability Process of Key Links in the Rice Industry Chain

\begin{tabular}{|c|c|c|c|}
\hline $\begin{array}{c}\text { Application } \\
\text { Layer }\end{array}$ & Registration and Login & Data Collection & Record Tracing \\
\hline $\begin{array}{c}\text { Interface } \\
\text { Layer }\end{array}$ & \multicolumn{3}{|c|}{ Web APIS } \\
\hline \multirow{2}{*}{$\begin{array}{c}\text { Service } \\
\text { Layer }\end{array}$} & $\mathrm{P} 2 \mathrm{P}$ & Consensus mechanism & Smart contract \\
\hline & & MQTT & Certificate Authority \\
\hline \multirow{2}{*}{$\begin{array}{c}\text { Physical } \\
\text { Layer }\end{array}$} & \multicolumn{3}{|c|}{ Consortium blockchain } \\
\hline & Mobile devices & Sensors & $\ldots$ \\
\hline
\end{tabular}

Figure 2. Rice Traceability System Architecture

- Warehousing node: Collect the data information in the rice storage link. It includes warehouse capacity, responsible person, and subordinate units;

- Processing node: Collect the data information in rice processing link. It includes product implementation standards, finished product names, ingredients, nutrients, quality, and shelf life.

- $\quad$ Sales node: Collects rice sales destination-related information.

The rice traceability system architecture includes four layers: the physical layer, service layer, interface layer, and application layer, as illustrated in Figure 2.

The physical layer includes consortium blockchain network, mobile devices and sensors. The consortium blockchain network consists of service node (the mainly functions include upload data, data query services, and data consensus verification to ensure data reliability in the system), orderer node (the mainly functions include manage the service node, generate new data-block, et al.), and certificate authentication (CA) node (It provides authentication function for system users or new node servers to access the system). Mobile devices (such as handheld code scanning terminal, mobile phone, monitor, et al.) and sensors (such as temperature sensor, humidity sensor, microbial sensor) are deployed in each link of rice supply chain to collect data.

The system service layer functions include P2P network construction that provides a solution to communicate among node servers of physical layer. The consensus function is used to ensure the consistency of each new data block in each node servers. Smart contracts are used to build digital contracts based on business contracts.

The system interface layer encapsulates service layer functions into standard web API interfaces, supporting external application access with business requirements.

The system application layer supports account registration and login. It provides a solution to automatically collect the information based on sensor equipment by docking its existing information system. Then the data would be distributed into the blockchain nodes. By scanning a QR code, consumers can access information from rice planting to retail. It is also helpful for enterprises in the rice supply chain to obtain the real quality information of rice raw materials and the actual market demand of their products. Which 


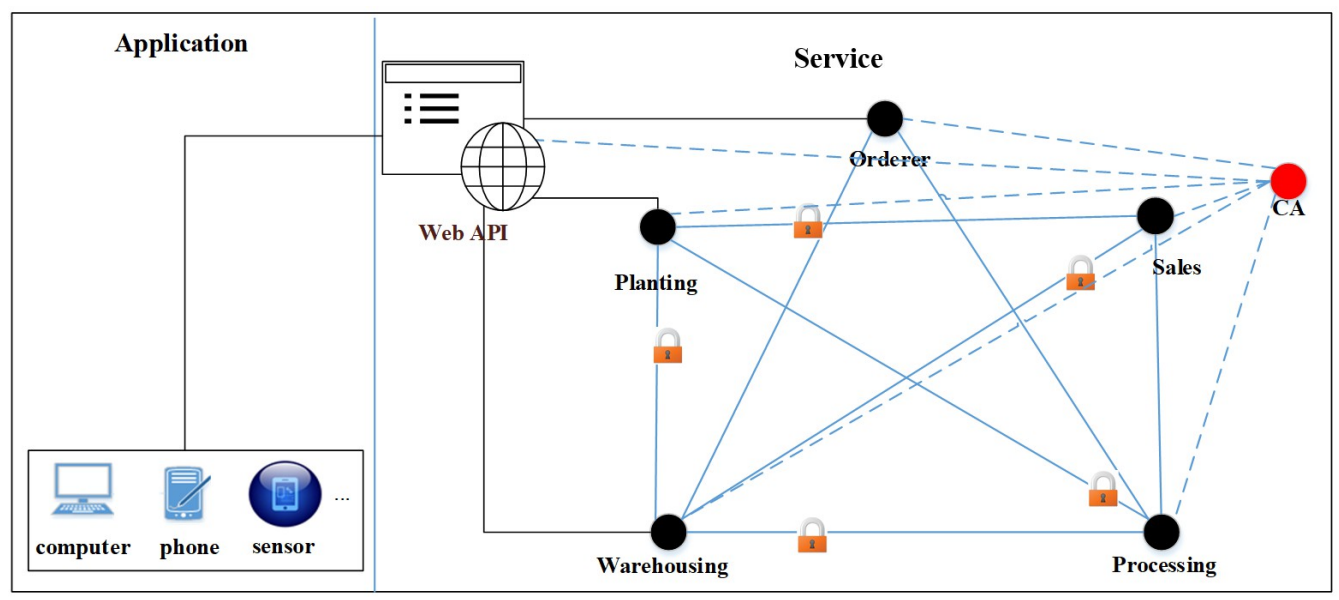

Figure 3. Network Structure of Rice Traceability System

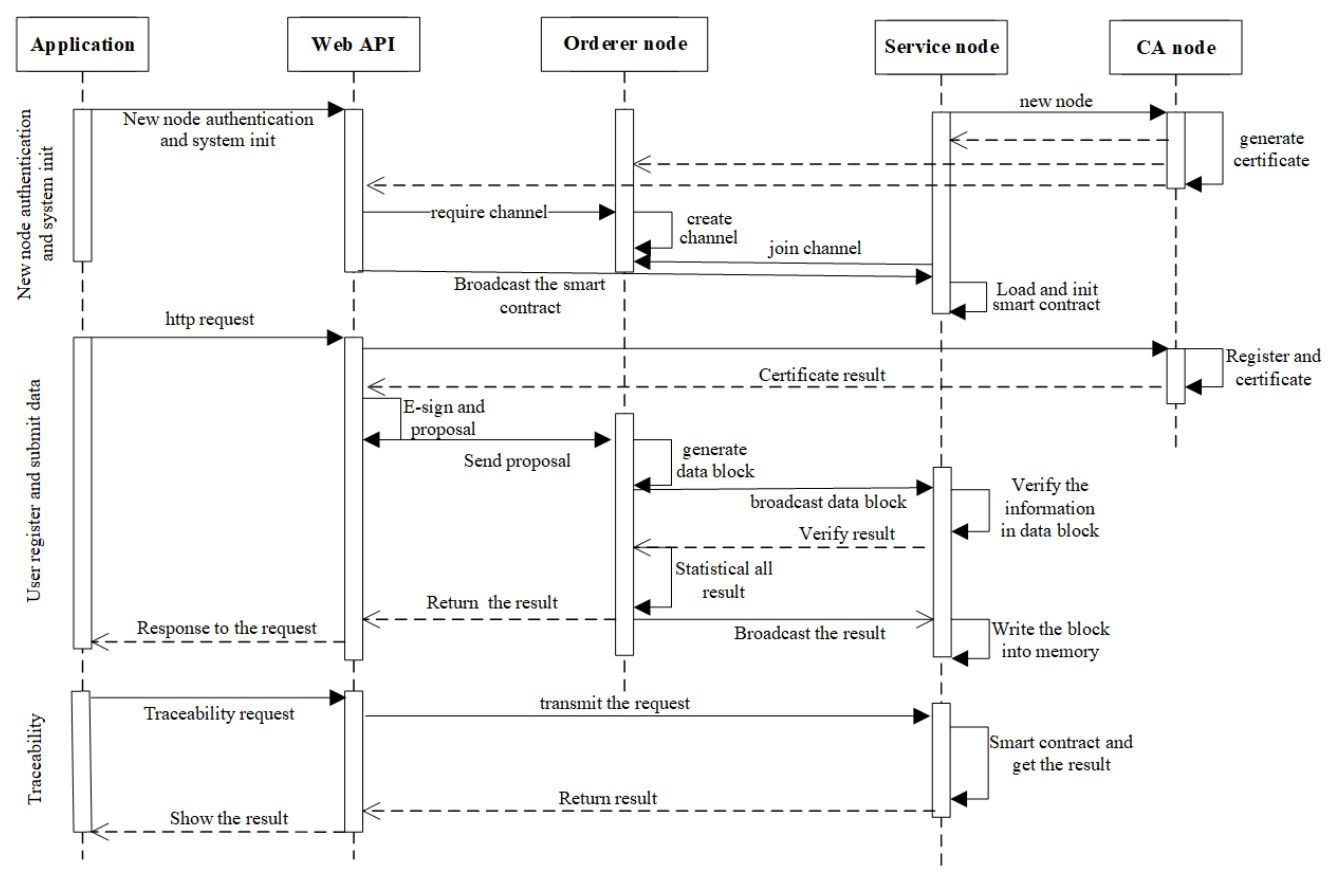

Figure 4. Sequence Diagram of Rice Traceability System

is meaningful for enterprises to optimize the rice industry chain in time, and avoid the food waste due to the backlog of rice product.

The system network architecture includes the application module and service module illustrated in Figure 3, which provides a solution to facilitate information interaction between modules by web API. The application module includes data sensor and mobile devices, which is mainly collecting data in each link of the rice supply chain, and shows the traceability results to consumers. The service module is a communication network of distributed nodes cluster based on consortium blockchain. It includes service nodes, orderer node, and CA node. Where service nodes provide the service to each link of rice industry such as planting, storage, processing, sales.. The orderer node is selected from service nodes based on consensus algorithm [25], which is used to encapsulate the collected data with chronological order into the blocks and organize all the service servers to verify the data records' authenticity. The CA node supports certificate issuance and management, issuing certificates, account registration, and certification for orderer nodes and business nodes.

The rice traceability system's overall functional flow illustrated in Figure 4 is detailed as follows. 
Table 1: System Configurations

\begin{tabular}{|c|c|c|c|c|}
\hline Server & System & CPU & Memory & IP \\
\hline $\begin{array}{l}\text { Orderer } \\
\text { Server }\end{array}$ & $\begin{array}{c}\text { Ubuntu } 16.04 \\
\text { 64bit }\end{array}$ & $\begin{array}{c}\text { Intel@ Xeon } \AA \text { CPU } \\
\text { E5-26xx v4 @2.3GHZ x } 1\end{array}$ & 2GB RAM & 119.29.11.57 \\
\hline $\begin{array}{l}\text { Service } \\
\text { Server } 1\end{array}$ & $\begin{array}{c}\text { Ubuntu } 16.04 \\
\text { 64bit }\end{array}$ & $\begin{array}{c}\text { Intel@ Xeon } \AA \text { CPU } \\
\text { E5-26xx v4 @2.3GHZ x } 1\end{array}$ & 2GB RAM & 119.29.11.57 \\
\hline $\begin{array}{l}\text { Service } \\
\text { Server } 2\end{array}$ & $\begin{array}{c}\text { Ubuntu } 16.04 \\
\text { 64bit }\end{array}$ & $\begin{array}{c}\text { Intel@ Xeon } \AA \text { CPU } \\
\text { E5-26xx v4 @2.3GHZ x } 1\end{array}$ & 2GB RAM & 119.29.150.15 \\
\hline CA Server & $\begin{array}{c}\text { Ubuntu } 16.04 \\
\text { 64bit }\end{array}$ & $\begin{array}{c}\text { Intel } \AA \text { core I7-4790K } \\
\text { CPU@4.00GHZ x } 8\end{array}$ & 8GB RAM & 192.168.152.144 \\
\hline Web API & $\begin{array}{c}\text { Ubuntu } 16.04 \\
\text { 64bit }\end{array}$ & $\begin{array}{c}\text { Intel@ core I7-4790K } \\
\text { CPU@4.00GHZ x } 8\end{array}$ & 8GB RAM & 192.168 .152 .250 \\
\hline
\end{tabular}

- The system initialization. The CA node server-generated public key certificates with different permission for each sever. Then the Orderer node server created a channel where the service node servers joined. The shared information was propagated in the channel, which avoided mixed data from different industrial chains. At the same time, the smart contract was deployed on each service node server.

- Response to the users' request. A new customer would register by the CA server and get a public certificate. The web API accepted the request and e-sign with the public certificate to construct a data proposal. The data proposal would then be sent to the Orderer server and be ordered with time sequence into the block in the orderer server. The block would be broadcast to the service servers, which would verify any data information in the block. The orderer server would get the final result by the results collected from service servers and broadcasted it. The service servers received and written the block memory.

- Response to the traceability request. The web API accepted the request and then transmit to one of the service servers with load balancing. The service server would trigger the smart contract to respond to the request and return the result.

\section{Implementation of Rice Traceability System}

The rice traceability system adopts the $\mathrm{B} / \mathrm{S}$ architecture mode. The technical architecture is Angular JS, Spring Boot, Hyperledger Fabric, CouchDB and Mysql database. The system server configuration information, as described in Table 1. The Orderer server and service servers are deployed on the external network and the CA server deployed on the Intranet. With the equipment restrictions, the Orderer server and service server deployed on the same server. However, it would have little influence on the system feasibility.

The application module uses the Angular JS to parse and display the information returned by the server. The server modules implement the blockchain business function based on Hyperledger Fabric V1.01 and encapsulate the fabric function module as a web API for the application module service call through Spring Boot. The service server nodes stored the transaction status with CouchDB. The MySQL database helps store each service information's tag information to achieve complex data-level logic processing.

The system simulates the collection, display, and traceability of information for the cultivation, warehousing, processing, and sales of the rice industry. Figure 5 and 6 shows the collection and record display of planting information. It supports the circulation of crops to the designated warehouse.

The traceability results for the sales records are shown in Figure 7. All information would be traceability step by step and showed to the customers, including the related sales information, processing information, warehousing information, and planting information. The system could track any downstream records until the planting information. 


Plant Warehouse Process Sale
< Records management $\quad$ New Record
Seed:
Plant area:

Fertilizer breed:

Fertilizing amount:

Pesticide breed:

Pesticide amount:

PH:

Water quality:

Figure 5. Collection of Planting Information

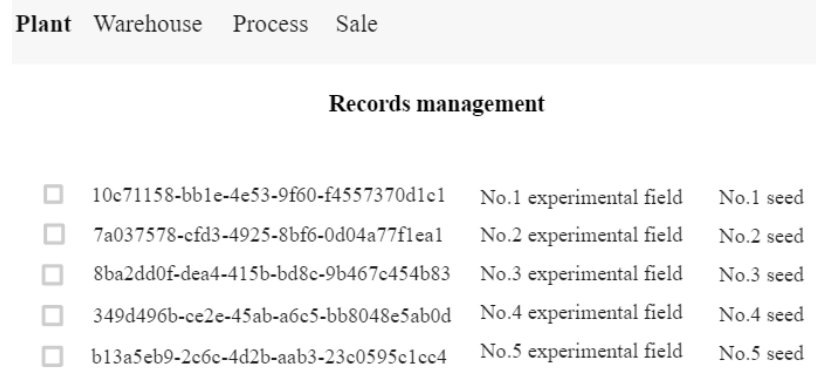

Select circulation enterprise: No.1 warehouse

Circulation submit

Figure 6. Planting Records and Circulation 


\begin{tabular}{ll}
\multicolumn{1}{c|}{ Back } & \\
\hline \multicolumn{1}{c|}{ Sale company: } & No.2 Wal-mart \\
\hline Location: & Wuhan, hubei province, China \\
\hline Linkman: & Alice \\
\hline Tel: & 15807052771 \\
\hline X company & $2 * *$ Street, Hongshan District, Wuhan City \\
\hline & 1
\end{tabular}

\begin{tabular}{l}
\hline \multicolumn{1}{c}{ Back } \\
\hline Process location: $\quad 2^{* *}$ Street, Hongshan District, Wuhan City \\
\hline Process company: X company \\
\hline Ingredients: $\quad$ water, rice \\
\hline Product standard: IDSOS856-1 \\
\hline
\end{tabular}

Responsible Person: Bob

\begin{tabular}{ll}
\hline Product: & Heilongjiang Wuchang rice flower \\
\hline Production date: & $2018-5-27$ \\
\hline Expiration date: & $2019-5-27$ \\
\hline Nutrients: & fiber, sugar \\
\hline Quality: & Level 1 \\
\hline Batch: & No.1 May, 2018 \\
\hline
\end{tabular}

\begin{tabular}{|l|l|}
\hline No.3 warehouse $15 \mathrm{t}$ \\
\hline \multicolumn{1}{c|}{ Back } \\
\hline Seed: & No.3 seed \\
\hline Plant area & $130 * *$ Street, Hongshan District, Wuhan City \\
\hline
\end{tabular}

Ingredients: water, rice

Fertilizer breed: $\quad$ Red Star organic fertilizer

\begin{tabular}{|l|l|}
\hline \multicolumn{1}{|c|}{ Back } & \\
\cline { 1 - 1 } Warehouse name: & No.3 warehouse \\
\hline Capacity: & $15 t$ \\
\hline Linkman: & Lucy \\
\hline Tel: & 18107052371 \\
\hline No.3 seed & 130 ** Street, Hongshan District, Wuhan City \\
\hline
\end{tabular}

Fertilizing amount: $100 \mathrm{mg} / \mathrm{m} 2$

Pesticide breed: $\quad$ Red Star pesticide

Pesticide amount: $\quad 10 \mathrm{mg} / \mathrm{m}^{2}$

PH:

Water quality:

Level 1

Figure 7. Trace Case of Sales Record 


\section{Conclusion and Future Work}

We proposed and implemented a supply chain traceability system based on blockchain technology. The system inherits characteristics such as tamper-proofing, decentralization and transparancy from blockchain technology. Our supply chain traceability system provides transparent and tamper-proof data through out the supply chain at every production step. It improves the product quality as well as data quality to compete into the consumer oriented market. The quality data can be used to assess the future production and quality control management. It will also promotes the healthy competion between the food producers to increase the product qualtiy to gain consumer trust and market share.

Recently, the system has realized the whole rice supply chain data sharing, but the ability of mining data value still needs to be improved. So, the further work is to establish a big data platform for rice supply chain based on the rice traceability system. And through the application of big data technology to achieve the healthy and sustainable development of the rice industry chain.

Author Contributions: Conceptualization, Tao Q., Iftekhar A.; Investigation, Iftekhar A.; Software, Cai Z.; Writing—original draft, Tao Q.; Writing—review and editing, Tao Q., Iftekhar A., and Cui X. All authors have read and agreed to the published version of the manuscript.

Funding: The authors would like to acknowledge the support provided by the National Key R\&D Program of China (No.2018YFC1604000).

Institutional Review Board Statement: Not applicable

Informed Consent Statement: Not applicable

Data Availability Statement: Data sharing is not applicable for this article.

Conflicts of Interest: The authors have no affiliation with any organization with a direct or indirect financial interest in the subject matter discussed in the manuscript.

\section{References}

1. Abraham, A.; Mathew, A.K.; Sindhu, R.; Pandey, A.; Binod, P. Potential of rice straw for bio-refining: An overview. Bioresource Technology 2016, 215, 29-36. doi:https://doi.org/10.1016/j.biortech.2016.04.011.

2. Haiyan, W.Y.G.B.Z.; Lei, W.S.Z. Research Pathway and the Application Principle of Food Tracing Technology [J]. Journal of Chinese Institute of Food Science and Technology 2012, 11.

3. Inácio, M.R.C.; de Moura, M.d.F.V.; de Lima, K.M.G. Classification and determination of total protein in milk powder using near infrared reflectance spectrometry and the successive projections algorithm for variable selection. Vibrational Spectroscopy 2011, $57,342-345$.

4. Boli, G.; Yimin, W.; Jiarong, P. Progress in the application of isotopic fingerprint analysis to food origin traceability [J]. Transactions of the Chinese Society of Agricultural Engineering 2007, 3.

5. Giancaspro, A.; Colasuonno, P.; Zito, D.; Blanco, A.; Pasqualone, A.; Gadaleta, A. Varietal traceability of bread 'Pane Nero di Castelvetrano' by denaturing high pressure liquid chromatography analysis of single nucleotide polymorphisms. Food Control 2016. doi:10.1016/j.foodcont.2015.07.006.

6. Montemurro, C.; Pasqualone, A.; Simeone, R.; Sabetta, W.; Blanco, A. AFLP molecular markers to identify virgin olive oils from single Italian cultivars. European Food Research and Technology 2008. doi:10.1007/s00217-007-0675-z.

7. Subrsmanian, V.; Chang, J.; Mattis, B.; Molesa, S.; Redinger, D.; De La Fuente Vornbrock, A.; Volkman, S.K. All-printed electronics: Materials, devices, and circuit implications. Digital Fabrication 2006 - Final Program and Proceedings, 2006.

8. Tao, Q.; Cui, X.; Huang, X.; Leigh, A.M.; Gu, H. Food Safety Supervision System Based on Hierarchical Multi-Domain Blockchain Network. IEEE Access 2019, 7, 51817-51826.

9. Iftekhar, A.; Cui, X. Blockchain-Based Traceability System That Ensures Food Safety Measures to Protect Consumer Safety and COVID-19 Free Supply Chains. Foods 2021, 10. doi:10.3390/foods10061289.

10. Bermeo-Almeida, O.; Cardenas-Rodriguez, M.; Samaniego-Cobo, T.; Ferruzola-Gómez, E.; Cabezas-Cabezas, R.; Bazán-Vera, W. Blockchain in Agriculture: A Systematic Literature Review. Technologies and Innovation; Valencia-García, R.; Alcaraz-Mármol, G.; Del Cioppo-Morstadt, J.; Vera-Lucio, N.; Bucaram-Leverone, M., Eds.; Springer International Publishing: Cham, 2018; pp. 44-56.

11. Tse, D.W.K.; Zhang, B.; Yang, Y.; Cheng, C.; Mu, H. Blockchain application in food supply information security 2017. pp. 1357-1361.

12. Schmidhuber, M.T. Emerging Opportunities for the Application of Blockchain in the Agri-food Industry 2018. 
13. Stadtler, H. Supply chain management and advanced planning - Basics, overview and challenges. European Journal of Operational Research 2005. doi:10.1016/j.ejor.2004.03.001.

14. Huckle, S.; White, M. Fake News: A Technological Approach to Proving the Origins of Content, Using Blockchains. Big Data 2017. doi:10.1089/big.2017.0071.

15. Kim, H.M.; Laskowski, M. Toward an ontology-driven blockchain design for supply-chain provenance. Intelligent Systems in Accounting, Finance and Management 2018. doi:10.1002/isaf.1424.

16. Seuring, S. A review of modeling approaches for sustainable supply chain management. Decision Support Systems, 2013. doi:10.1016/j.dss.2012.05.053.

17. Storoy, J.; Thakur, M.; Olsen, P. The TraceFood Framework - Principles and guidelines for implementing traceability in food value chains. Journal of Food Engineering 2013. doi:10.1016/j.jfoodeng.2012.09.018.

18. Iftekhar, A.; Cui, X.; Hassan, M.; Afzal, W. Application of Blockchain and Internet of Things to Ensure Tamper-Proof Data Availability for Food Safety. Journal of Food Quality 2020. doi:10.1155/2020/5385207.

19. Sun Zhiguo, Li Xiufeng, W.W.J.Z. Application Prospect of Block Chain Technology in Food Safety. Agriculture Network Information 2016.

20. Cocco, L.; Pinna, A.; Marchesi, M. Banking on blockchain: Costs savings thanks to the blockchain technology. Future Internet 2017. doi:10.3390/fi9030025.

21. Garaus, M.; Treiblmaier, H. The influence of blockchain-based food traceability on retailer choice: The mediating role of trust. Food Control 2021, p. 108082. doi:https://doi.org/10.1016/j.foodcont.2021.108082.

22. Q Tao, Q Chen, H.D.I.A.X.H.; Cui, X. Cross-Department Secures Data Sharing in Food Industry via Blockchain-Cloud Fusion Scheme. Security and Communication Networks 2021, 2021, 1-18. doi:https:/ / doi.org/10.1155/2021/6668339.

23. The Blockchain Technology and Application Development Whitepaper. Department of Informatization and Software Services, Ministry of Industry and Information Technology 2016.

24. Handbook for Introduction of Food Traceability Systems. Tokyo Food Marketing Research and Information Center 2007.

25. Zhang, P.; Schmidt, D.C.; White, J.; Dubey, A. Chapter Seven - Consensus mechanisms and information security technologies. In Role of Blockchain Technology in IoT Applications; Elsevier, 2019; Vol. 115, pp. 181-209. doi:https://doi.org/10.1016/bs.adcom.2019.05.001. 